\title{
Mediation effect of obesity on the association between triglyceride-glucose index and hyperuricemia in Chinese hypertension adults.
}

Jin Sun

Medical School of Chinese PLA: Chinese PLA General Hospital

Yongkang Su

Medical School of Chinese PLA: Chinese PLA General Hospital

Man Li

Medical School of Chinese PLA: Chinese PLA General Hospital

Shouyuan $\mathrm{Ma}$

Chinese PLA General Hospital

Yan Zhang

Chinese PLA General Hospital

Anhang Zhang

Medical School of Chinese PLA: Chinese PLA General Hospital

Shuang Cai

Medical School of Chinese PLA: Chinese PLA General Hospital

Bokai Cheng

Medical School of Chinese PLA: Chinese PLA General Hospital

\section{Qiligeer Bao}

Medical School of Chinese PLA: Chinese PLA General Hospital

Shuxia Wang

Chinese PLA General Hospital

Ping Zhu ( $\square$ zhup301@163.com )

PLAGH: Chinese PLA General Hospital

Original investigation

Keywords: Triglyceride-glucose index, Hyperuricemia, Obesity, Hypertension, Mediation effect

Posted Date: September 23rd, 2021

DOI: https://doi.org/10.21203/rs.3.rs-779315/v2 
License: (c) (i) This work is licensed under a Creative Commons Attribution 4.0 International License. Read Full License

Version of Record: A version of this preprint was published at The Journal of Clinical Hypertension on December 13th, 2021. See the published version at https://doi.org/10.1111/jch.14405. 


\section{Abstract}

Background and Aims: The triglyceride glucose (TyG) index was regarded as a simple surrogate marker of insulin resistance (IR). It is confirmed that IR was significantly associated with hyperuricemia, and obesity was the risk factor for IR and hyperuricemia. However, the relationship between the TyG index and hyperuricemia and the potential role of obesity in Han Chinese hypertension are not entirely elucidated.

Methods and Results $\rrbracket$ A community-based cross-sectional study was conducted in 4551 hypertension patients aged 40-75 years with clinical and biochemical data. The TyG index was calculated as In [fasting triglyceride $(\mathrm{mg} / \mathrm{dl}) \times$ fasting blood glucose $(\mathrm{mg} / \mathrm{dl}) / 2$ ]. Hyperuricemia was determined as serum uric acid $\geq 357 \mu \mathrm{mol} / \mathrm{L}(6 \mathrm{mg} / \mathrm{dl})$ for females and $\geq 417 \mu \mathrm{mol} / \mathrm{L}(7 \mathrm{mg} / \mathrm{dl})$ for males. The TyG index was higher in patients with hyperuricemia than in those without $(8.99 \pm 0.61,8.70 \pm 0.59, \mathrm{P}<0.001)$. The prevalence of hyperuricemia in patients with the lowest $(\leq 8.32)$, second (8.33-8.66), third (8.67-9.07) and the highest quartile $(\geq 9.08)$ of the TyG index was $6.0 \%, 10.4 \%, 15.4 \%, 21.4 \%$, respectively. Logistic regression analysis suggested that the higher quartile of TyG index was associated with increased hyperuricemia risk whether in crude or adjusted models $(P<0.05)$. Mediation analysis showed that all of our obesity indexes partially mediated the association between the TyG index and hyperuricemia to some extent.

Conclusion囚TyG index is significantly associated with hyperuricemia in hypertension patients among Han Chinese, obesity plays a partial mediation role in this relationship.

\section{Introduction}

Hyperuricemia is caused by abnormal purine metabolism, including excessive uric acid production or insufficient renal excretion, which is one of the components of metabolic syndrome. Previous studies have shown that hyperuricemia is associated with the occurrence and development of many metabolic disorders and cardiovascular diseases. For example, hypertensive patients with hyperuricemia occurred more cardiovascular events than those without hyperuricemia ${ }^{[1,2]}$. Recently epidemiological study reported that there are 170 million patients with hyperuricemia in China ${ }^{[3]}$, which greatly increases the morbidity and mortality of cardiovascular events. Therefore, it is very important to optimize the risk stratification method of hyperuricemia to identify people at high risk of cardiovascular events and other complications. Some studies have reported that IR and obesity are independent risk factors for hyperuricemia, which are significantly related to the occurrence and development of hyperuricemia ${ }^{[4,5]}$.

It is recognized that the bidirectional correlation between IR and hyperuricemia ${ }^{[6,7]}$. The increase of uric acid level will lead to the impairment of endothelial function, which in turn reduces insulin sensitivity by reducing the bioavailability of nitric oxide, and eventually leads to $\mathbb{I}^{[8,9]}$. In contrast, $I R$ induces hyperuricemia by increasing the reabsorption of uric acid and sodium in renal tubules ${ }^{[10]}$. Some epidemiological studies have also confirmed the two side effect of IR and hyperuricemia. A longitudinal study reported the unidirectional association between the two. They found that hyperuricemia leads to IR, then partially mediates the development of hypertension ${ }^{[11]}$. But another study found that the 
improvement of insulin sensitivity reduces the level of serum uric acid ${ }^{[12]}$. In short, IR and hyperuricemia promote each other to form a vicious circle, resulting in more serious organ damage. In addition, some studies confirmed that IR is related to obesity ${ }^{[13]}$, and obesity is a major risk factor for hyperuricemia ${ }^{[14]}$, so the correlation between IR and hyperuricemia may be partially or completely mediated by obesity.

At present, the TyG index is considered as an alternative index to identify $\mathbb{I R}^{[15]}$, and it is confirmed that the correlation with the occurrence and prognosis of many IR-related diseases ${ }^{[16]}$. It has been reported that there is a positive correlation between the TyG index and the risk of hyperuricemia ${ }^{[17-19]}$, and the IR was more significant in patients with hypertension complicated with hyperuricemia. However, most of these studies were conducted in relatively healthy communities. The role of the TyG index in assessing the risk of hyperuricemia in hypertensive patients and the potential mechanism of obesity in it have not been fully clarified. Therefore, the purpose of our study is to explore the relationship between TyG index and the prevalence of hyperuricemia in the hypertensive population, and further to clarify whether obesity indexes $(\mathrm{BMI}, \mathrm{WC}, \mathrm{HC})$ play an intermediary role in it, to optimize the risk stratification method of hyperuricemia and further identify people at very high risk of cardiovascular events and other complications in a hypertensive population.

\section{Methods}

\section{Study population:}

This was a community-based cross-sectional study, and participants were recruited from the Xinyang county, in the middle region in China from 2004-2005. We used a multistage cluster sample method to select a representative sample of rural community residents aged $40-75$ years. A total of 13444 subjects (5270 men and 8174 women) were incorporated into the survey, which was from 63 districts of Xinyang's seven residential communities and yielding a response rate of $84.9 \%$. Among them, 5421 hypertensive patients were identified and thoroughly examined. Hypertension was defined as diastolic blood pressure (DBP) of $\geq 90 \mathrm{mmHg}$, SBP of $\geq 140 \mathrm{mmHg}$, physician diagnosis, or current medication for hypertension (as defined by WHO 1999). Of 5421 hypertensive patients, 4805 patients had complete echocardiographic data, and 254 subjects were excluded because of no data about other clinical characteristics or blood biochemical indexes. Ultimately, 4551 patients (1531 men and 3020 women) with integrated clinical data remained in the present study.

\section{Clinical characteristics:}

Based on the age on the residence documents, we identified those who are eligible participants, then we invited them by letter or phone to the community clinic. All the participants were interviewed and required to complete a standardized questionnaire that included general information, such as sex, age, medical history, lifestyle behaviors, and so on. Anthropometric measurement was performed by experienced research staff with uniform instruments. The height and weight of all participants were measured in the upright position with light clothing and bare feet, and the error range is not more than $0.1 \mathrm{~cm}$ or $0.1 \mathrm{~kg}$. We 
measured waist and hip measurements for all participants, also while standing. The Blood pressure was measured using a standard official protocol. Systolic and diastolic blood pressures (SBP, DBP) were measured using the portable Doppler device (ES-101EX, HADECO, $8 \mathrm{MHz}$ probes, Kawasaki, Japan) and standard 12-cm cuff in each arm with the patients resting for at least $5 \mathrm{~min}$. The Doppler Stethoscope was placed at the humeral artery fluctuation, quickly inflate the cuff to $20-30 \mathrm{mmHg}$ above the palpated SBP, and then deflated at the rate of $2-6 \mathrm{mmHg} / \mathrm{s}$. The first sound heard is the SBP of the brachial artery, which continues to deflate until the sound disappears or suddenly becomes weak, the scale indicated by the mercury column is DBP. The average of three readings with the participant in the sitting position after at least $5 \mathrm{~min}$ of rest, recorded at least 30 s apart, was obtained for analysis.

Transthoracic echocardiography was performed according to standard protocols, under the supervision of two ultrasound physicians with at least two years of experience, and performed by two technicians trained in echocardiography at the Institute of Cardiology, Chinese Academy of Medical Sciences. The patients respiring quietly in the left decubitus position, and the echocardiographic indicators were measured at the end of systolic and end-diastolic periods of up to three cardiac cycles, including left atrium diameter, diastolic left ventricular inner diameter (LVIDD), diastolic left ventricular posterior wall thickness (PWTd), diastolic interventricular septal thickness (IVSd), E wave deceleration time, transmitted E wave velocity and transmitted $A$ wave velocity. Then we calculated the left ventricular mass (LVM) and left ventricular mass index (LVMI) based on the above data. LVM was calculated by using the equation: $0.8 \times 1.04\left((\text { IVSd + LVIDD + PWTd })^{3}-\right.$ LVIDD $\left.^{3}\right)+0.6$. LVMI was calculated by dividing LVM by height $^{2.7}\left(\mathrm{LVMI}_{\mathrm{h} 2.7}\right)$.

$\mathrm{BMI}$ was calculated as the ratio of the weight in $\mathrm{kg}$ divided by the square of the height in $\mathrm{m}$. The diagnosis of diabetes mellitus (DM) was based on an increased fasting plasma glucose $(\geq 7.0 \mathrm{mmol} / \mathrm{L})$, previous physician diagnosis, or current anti-diabetic medication. The diagnosis of stroke was based on the results of strict neurological examination, computed tomography, or magnetic resonance imaging tests, which were verified from local hospital records. Coronary heart disease (CAD) was diagnosed by the results of coronary arteriography, a previous myocardial infarction, or surgery or coronary revascularization.

\section{Biochemical parameters:}

Fasting blood samples were obtained from an antecubital vein of participants after overnight fasting. Serum was separated from the blood samples by centrifuged on-site. Then the serum samples were delivered to the Beijing center laboratory on the dry ice for analysis. The fasting blood glucose (FPG), triglycerides (TG), total cholesterol (TC), high-density lipoprotein (HDL) cholesterol, low-density lipoprotein (LDL) cholesterol, uric acid, and other blood biochemical indices were quantified enzymatically by an automatic analyzer (Hitachi 7060, Hitachi, Tokyo, Japan).

The TyG index was calculated as In [fasting TG $(\mathrm{mg} / \mathrm{dl}) \times \mathrm{FPG}(\mathrm{mg} / \mathrm{dl}) / 2]^{[20]}$. Patients were divided into four groups according to the TyG index quartile: Q1 (TyG $\leq 8.32)$, Q2 (8.33 $\leq$ TyG $\leq 8.66)$, Q3 
(8.67 $\leq \mathrm{TyG} \leq 9.07$ ), and Q4 ( $\geq 9.08)$. Hyperuricemia was determined as serum uric acid $\geq 357 \mu \mathrm{mol} / \mathrm{L}$ (6 $\mathrm{mg} / \mathrm{dl})$ for females and $\geq 417 \mu \mathrm{mol} / \mathrm{L}(7 \mathrm{mg} / \mathrm{dl})$ for males ${ }^{[21]}$.

\section{Statistical analysis:}

Data management and statistical analysis were performed using SPSS 22.0 for Windows (SPSS Inc, Chicago, IL, USA). Data are reported as the mean \pm standard deviation for continuous variables and as percentages for categorical variables. Continuous variable independent sample t-test and classified variable chi-square test were used for the differences between hyperuricemia group and nonhyperuricemia group. All participants were stratified by quartiles of TyG index, baseline differences in clinical variables between groups using analysis of variance (ANOVA) for continuous variables, and Chisquared test for categorical variables. We used the multivariable-adjusted logistic regression model to evaluate the relationship between TyG index categories and hyperuricemia, and the values of odds ratios (ORs) and 95\% confidence intervals (Cls) were calculated. Sequential models were developed to minimize the effect of confounders. Model 1 was a crude model. Model 2 was adjusted for age, sex, SBP, DBP, serum creatinine, blood urea nitrogen (BUN), the history of stroke, CAD, and DM. Model 3 was adjusted for all variables of model 2 plus serum TC, HDL-C, LDL-C BMI, HC, and WC.

To further examine the impact of BMI, WC, and $\mathrm{HC}$ on the association between TyG index and hyperuricemia, we constructed mediation models for analysis. In the model, TyG index is predictor, $\mathrm{BMI}$, $\mathrm{HC}$, and WC are mediators respectively, uric acid is the outcome, and the confounders in model 3 were adjusted in the mediation analysis. The simplified mediation model is presented in Fig 1, which involved several main paths as follows. The path a: the association between TyG index and obesity indexes; Path b: the association of obesity indexes with uric acid; Path $c$ and Path $c^{\prime}$ : the total and direct effects of TyG index on uric acid, respectively. In addition, Path ab mains the indirect effect of the TyG index on uric acid, and the sum of direct and indirect effects equals the total effect. The obesity indexes played a complete mediating role in the association between the TyG index and uric acid when the total effect and indirect effect are significant but the direct effect is not significant. However, the obesity indexes were the incomplete mediator when the direct effect is also significant. The differences were considered significant if a 2-tailed $P$ value $<0.05$.

\section{Results}

\section{Clinical characteristics of patients by hyperuricemia}

In the whole group of 4551 hypertension patients, there were 605 patients with hyperuricemia, the prevalence of hyperuricemia was $13.29 \%$. The clinical characteristics of the study population by hyperuricemia are described in Table 1. The average age of all participants was $58.63 \pm 8.33$ years, of which patients with hyperuricemia were $60.11 \pm 8.76$ years, and it was $58.41 \pm 8.24$ years in people without hyperuricemia. Males comprised $33.6 \%$ of the total participants, $51.2 \%$ of the hyperuricemia group, and $30.9 \%$ of the non-hyperuricemia group. Compared to subjects without hyperuricemia, those with hyperuricemia were more likely to be older, with a higher proportion of males, had higher weight, height, 
BMI, WC, HC, serum TG, TC, HDL-C, LDL-C, uric acid, BUN, and creatinine, and more CAD status and stroke history (all P凶0.05). In addition, Echocardiographic indicators (IVSd, PWTd, LVM, and LVMI) were significantly higher in the hyperuricemia group than in the non-hyperuricemia group. Particularly worth mentioning is the TyG index is significantly higher in patients with hyperuricemia than without it $(8.99 \pm 0.61,8.70 \pm 0.59, \mathrm{P} \otimes 0.001)$.

\section{Clinical characteristics of patients by TyG index}

Based on the above results, the TyG index was grouped by quartile, and we presented the baseline characteristics of the subjects in Table 2 according to the TyG index categorical. In all participants, the prevalence of hyperuricemia with the lowest, second, third, and highest quartile of TyG index were 6.0, $10.4,15.4,21.4 \%$, respectively. It means an increasing trend in the prevalence of hyperuricemia with the higher TyG index (P凶0.001). Compared with patients with the lowest quartiles of TyG index, those with higher quartile of TyG index had higher weight, BMI, WC, HC, SBP, DBP, heart rate, and more CAD and DM status (all $\mathrm{P} \otimes 0.001$ ). In the biochemical index results, the group with a higher TyG index had higher serum FPG, TG, TC, HDL-C, uric acid, and lower BUN, LDL-C (all P®0.001). There were no significant differences in age, height, serum creatinine, or the morbidity of stroke among those groups.

\section{Association of TyG index with hyperuricemia}

To explore the relationship between the TyG index and hyperuricemia, we built logistics regression models, and the results were presented in Table 3. Our study revealed a positive correlation between the TyG index and hyperuricemia. As shown in Table 3, participants with the second, third, and highest quartile of TyG index were 1.83, 2.87, and 4.30 times more likely to have hyperuricemia compared with the lowest quartile in the crude model (P®0.001). After controlling for age, sex, SBP, DBP, serum creatinine, BUN, the history of stroke, CAD, and DM in model 2, the OR $(95 \% \mathrm{Cl})$ for hyperuricemia by TyG index groups was changed to 1.93 (1.38-2.70), 3.61 (2.62-4.97) and 6.47 (4.70-8.91), respectively. The relationship between TyG index and hyperuricemia remained significant after further adjustment for serum TC, HDL-C, LDL-C, BMI, WC, and $\mathrm{HC}$ in model 3 , and the OR $(95 \% \mathrm{Cl})$ of second, third, and highest quartile of TyG index compared with the lowest quartile were 1.60 (1.14-2.26), 2.47 (1.76-3.48) and 3.45 (2.35-5.07), respectively. In particular, the association between the TyG index and hyperuricemia attenuated after being adjusted for several biochemical markers and BMI but remained significant.

\section{Association of TyG index with obesity indexes and serum uric acid}

Table 4 shows the associations of the TyG index with obesity indexes (BMI, WC, and $\mathrm{HC}$ ) and serum uric acid. The $\beta$ coefficients $(95 \% \mathrm{Cl})$ of second, third, highest quartile of TyG index with BMI were $0.17(0.09$ $0.25), 0.32(0.24-0.40)$, and $0.47(0.38-0.55)$, respectively $(P<0.001)$ after adjusting for age, sex, SBP, DBP, serum creatinine, BUN, the history of stroke, CAD and DM. Similarly. TyG index was significantly positively correlated with WC and HC. In addition, the relationship between the TyG index and serum uric acid level is also statistically significant. Compared to the lowest quartile of the TyG index, the $\beta$ coefficients (95\% 
$\mathrm{Cl}$ ) of the second, third, highest quartile of TyG index with uric acid were increased gradually, which are $0.26,0.47,0.73$, respectively $(P<0.001)$.

\section{The mediating role of obesity indexes}

To further examine the impact of obesity indexes on the association between the TyG index and hyperuricemia, we constructed a mediation model to analyze. The results of mediation analysis were displayed in Table 5 and the simplified mediation model was presented in Figure 1. We found that all of our interested obesity indexes had a mediation impact (to various extents) on the link between the TyG index and uric acid. The total effect TyG index on uric acid was significant (total effect, $\beta(95 \% \mathrm{Cl}): 0.2430$ (0.2041-0.2818); $\mathrm{P} \otimes 0.001)$. And the proportion of the mediation varied from 7.3, 10.2, and 16.7 for $\mathrm{BMI}$, $\mathrm{HC}$, and WC, respectively. In conclusion, our study found that obesity indexes, including BMI, WC, and HC, partially mediated the association of the TyG index and uric acid as mediator factors.

\section{Discussion}

In this study, we observed that a strong cross-sectional correlation between the higher TyG index and the increased prevalence of hyperuricemia in Chinese patients with hypertension, which was statistically significant even after adjusting for clinical data and risk factors for hyperuricemia. We further evaluated the mediation effect of different obesity factors on the link of TyG index and serum uric acid level, we found that higher TyG index was associated with higher obesity index (BMI, WC, HC) and serum uric acid level, while the obesity factors partially mediated the relationship between TyG index and uric acid to a varied extent.

Hyperuricemia is a common metabolic abnormality and an independent risk factor for cardiovascular disease $^{[22]}$. A meta-analysis of 44 studies showed that the total prevalence of hyperuricemia in China was $13.3 \%{ }^{[21]}$, which was almost consistent with our results. However, previous studies have reported that the prevalence of hyperuricemia in hypertensive people is higher than that in normal people ${ }^{[23]}$. Our study was included hypertensive people, but the prevalence was similar to that in normal people. We assumed that may be due to the deviation of the sex ratio of our participants. It is reported that the prevalence of hyperuricemia in males is significantly higher than that in females $(19.4 \% \mathrm{vs} 7.9 \%)^{[21]}$, and our participants are far fewer in males than in females.

Hypertensive patients with hyperuricemia have a very high risk of developing cardiovascular disease ${ }^{[24]}$. A large number of cross-sectional and prospective studies have shown that high levels of uric acid increased the risk of target organ damage and cardiovascular events in patients with hypertension ${ }^{[25,26]}$. In our study, the prevalence of stroke and CAD in the hyperuricemia group was significantly higher than that in the normal uric acid group, and the significant differences in echocardiographic indicators between the two groups also indicated the damage of high uric acid levels to the heart. The potential mechanism involves many aspects. The decrease of renal blood flow in patients with hypertension leads to the increase of uric acid reabsorption, and the increase of high uric acid levels leads to the storage and 
flow of water and sodium in the body, which further increases hypertension, which is forming a small vicious circle. Hypertension is the most important risk factor for cardiovascular disease due to its damage to the function and structure of blood vessels ${ }^{[27]}$, while hyperuricemia leads to arterial stiffness by increasing the level of oxidative stress and inflammation in the body. The synergistic effect of the two accelerates the occurrence of cardiovascular complications ${ }^{[28,29]}$. Therefore, looking for new biological indicators to identify hyperuricemia in patients with hypertension is very important for the early prevention and intervention of cardiovascular complications.

IR plays a key role in the occurrence and development of hyperuricemia. As a new, simple but effective substitute for IR, the TyG index has been proved to be associated with a variety of cardiovascular diseases and related risk factors, including peripheral vascular disease, ischemic heart disease, coronary artery calcification, and arterial stiffness ${ }^{[30-32]}$. Previous studies reported that the TyG index may also be used as a risk index for hyperuricemia, and our results were supported by it. A cross-sectional study of the Chinese general population reported that the risk of hyperuricemia increases proportionally with the increase of the TyG index. They think the TyG index can significantly improve the risk identification ability of the risk prediction model for hyperuricemia, suggesting its important value in optimizing the risk stratification of hyperuricemia ${ }^{[17]}$. Recently, Li et al found that the correlation between TyG index and the risk of hypertension with hyperuricemia is more significant than that with hyperuricemia or hypertension alone, suggesting that IR is more significant in patients with hyperuricemia with hypertension ${ }^{[19]}$. Mazidi et al also reported a significant correlation between serum uric acid levels and IR in general communities of the United States, and they further found that a variety of obesity indicators mediated this correlation in varying degrees ${ }^{[18]}$.

In addition, some studies have suggested that obesity is a prerequisite for metabolic syndrome, and IR, hyperuricemia, and hypertension are all manifestations of metabolic syndrome ${ }^{[33]}$. Unfortunately, no studies have reported the effect of obesity on TyG index and hyperuricemia in hypertensive people, and previous studies on the role of obesity in the link between TyG index and hyperuricemia are inconsistent. In 2017, Mazidi et al reported that a variety of obesity indicators mediated the relationship between TyG and serum uric acid to varying degrees, of which the regulatory ratio of BMI to WC was $46.8 \%$ and $57.1 \%$, respectively ${ }^{[18]}$. However, a recent study reports that the link between the TyG index and hyperuricemia is not related to the mediation of BMI in the general community population in China ${ }^{[17]}$. Our study found that BMI, WC, and HC did partially mediate the relationship between TyG and serum uric acid, but the intermediary proportion was significantly lower than that of the NHANES study. We speculate that the reason for this difference may be due to ethnic differences and medical history differences in the included population. Previous studies have been reported that race affects insulin sensitivity and obesityrelated diseases ${ }^{[34]}$, while the incidence of overweight and IR in patients with hypertension is significantly higher than that in healthy people ${ }^{[35]}$, suggesting that IR and obesity indicators are also affected by hypertension. Perhaps for the above reasons, our study shows that the intermediary role of obesity in Chinese hypertensive people, which is different from that of the general population in China and weaker than that of the American population. 
The incidence of cardiovascular events and metabolic diseases in patients with hyperuricemia is significantly higher than that in healthy people, and hypertension is a common risk factor and complication of hyperuricemia. At present, there are 245 million people with hypertension and 170 million patients with hyperuricemia in China. Early identification of high-risk groups of hyperuricemia in patients with hypertension and early intervention for patients with complications is very important for the prevention and treatment of cardiovascular disease. Our study observed that a higher TyG index identifies people with hyperuricemia in the hypertension group, which is also at high risk of cardiovascular events, and contributes to the development of appropriate prevention and intervention strategies.

This study has several limitations to consider. First of all, our study only included the hypertensive population of the Han nationality in rural China, which could not represent the urban population. Second, the proportion of men and women included in this study is uneven, and men are far less than women, which may be because a large number of male rural residents leave home to work in cities. However, previous studies have reported that the incidence of hyperuricemia in men is significantly higher than that in women ${ }^{[21]}$, so follow-up studies with a more balanced sex ratio may be needed to prove our point of view. Third, we did not obtain data on uric acid drug use due to the design of the early study, but taking into account the regional economic situation and the general drug use in that period and the absence of anti-uric acid agents in previous studies, we think our results are reliable and can be compared with other studies. Finally, this is a cross-sectional study, so that we can only observe the correlation between the TyG index, hyperuricemia, and obesity indicators, but not know their time sequence. More rigorous prospective studies are needed to support our conclusions.

\section{Conclusion}

Our results show that a higher TyG index is significantly associated with the prevalence of hyperuricemia in hypertensive people. We further proved this link may be partially mediated by obesity and discussed the differences in the mediating effects of obesity in different populations. Therefore, the TyG index can not only be used as a predictor of hyperuricemia but also identify high-risk subjects in people with traditional risk factors, because it can further identify very high-risk groups in patients with hypertension. Our study quantifies the role of obesity in the association between TyG index and uric acid, and the control of obesity may contribute to the primary prevention of IR and hyperuricemia. In summary, we recommend routine determination of the TyG index in hypertensive people, which is a simple, low-cost but very beneficial test. In addition to screening patients with $I R$, it is also helpful to identify hypertensive people with hyperuricemia, who with a high risk of cardiovascular complications.

\section{Declarations}

Fund: This study was supported by the "National Key R\&D Program of China" (Funding No.2020YFC2008900), co-funded by the Military Medical Youth Growth Project of PLA General Hospital (Funding No. QNC19005), National defense science and technology innovation projects (Funding No. 19163-15-ZD-009-001-10). 
Conflict of interest: All the authors declared that they have no conflict of interest.

Availability of data and materialn The research data used to support the finding of this study are available from the corresponding authors upon request.

Code availabilityn Not applicable.

Authors' contributions: JS contributed to data interpretation, critical review of the manuscript, drafting the manuscript, and revising the comment of reviewers. YS contributed to manuscript preparation, analysis tools and assist with data analysis. $\mathrm{ML}, \mathrm{SM}$, and $\mathrm{YZ}$ contributed to manuscript revisions. $\mathrm{AZ}, \mathrm{SC}, \mathrm{BC}$, and QB helped with data analysis. SW contributed significantly to data collection, the conception of the study, and revising the comment of reviewers. PZ contributed to the conception of the study and helped perform the analysis with constructive discussions.

Acknowledgments: Not applicable.

Ethics approval $\square$ The study protocol was reviewed and approved by the ethical committees of the Fuwai Hospital and local hospitals. All participants gave their informed consent before they were recruited and reported themselves to be Han people.

Consent to participate】 Not applicable.

Consent for publication! Not applicable.

\section{Abbreviations}

BMI: body mass index; BUN: blood urea nitrogen; CAD: coronary heart disease; Cl: confidence interval; DBP: diastolic blood pressure; DM: diabetes mellitus; FPG: fasting blood-glucose; HC: hip circumference; HDL: high-density lipoprotein cholesterol; IR: insulin resistance; IVSd: diastolic interventricular septal thickness; LDL: low-density lipoprotein cholesterol; LVIDD: diastolic left ventricular inner diameter; LVM: left ventricular mass; LVMI: left ventricular mass index; OR: odds ratio; PWTd: posterior wall thickness; SBP: systolic blood pressure; TC: total cholesterol; TG: triglyceride; TyG index: triglyceride glucose index; WC: waist circumference

\section{References}

[1] Fang J, Alderman M H. Serum uric acid and cardiovascular mortality the NHANES I epidemiologic follow-up study, 1971-1992. National Health and Nutrition Examination Survey [J]. Jama, 2000, 283(18): 2404-10.

[2] Virdis A, Masi S, Casiglia E, Tikhonoff V, Cicero A F G, Ungar A, Rivasi G, Salvetti M, Barbagallo C M, Bombelli M, Dell'Oro R, Bruno B, Lippa L, D'Elia L, Verdecchia P, Mallamaci F, Cirillo M, Rattazzi M, Cirillo P, Gesualdo L, Mazza A, Giannattasio C, Maloberti A, Volpe M, Tocci G, Georgiopoulos G, laccarino 
G, Nazzaro P, Parati G, Palatini P, Galletti F, Ferri C, Desideri G, Viazzi F, Pontremoli R, Muiesan M L, Grassi G, Borghi C. Identification of the Uric Acid Thresholds Predicting an Increased Total and Cardiovascular Mortality Over 20 Years [J]. Hypertension (Dallas, Tex : 1979), 2020, 75(2): 302-8.

[3] Hao Y, Li H, Cao Y, Chen Y, Lei M, Zhang T, Xiao Y, Chu B, Qian Z. Uricase and Horseradish Peroxidase Hybrid CaHPO囚 Nanoflower Integrated with Transcutaneous Patches for Treatment of Hyperuricemia [J]. Journal of biomedical nanotechnology, 2019, 15(5): 951-65.

[4] Adnan E, Rahman I A, Faridin H P. Relationship between insulin resistance, metabolic syndrome components and serum uric acid [J]. Diabetes \& metabolic syndrome, 2019, 13(3): 2158-62.

[5] Gong M, Wen S, Nguyen T, Wang C, Jin J, Zhou L. Converging Relationships of Obesity and Hyperuricemia with Special Reference to Metabolic Disorders and Plausible Therapeutic Implications [J]. Diabetes, metabolic syndrome and obesity : targets and therapy, 2020, 13(943-62.

[6] Facchini F, Chen Y D, Hollenbeck C B, Reaven G M. Relationship between resistance to insulinmediated glucose uptake, urinary uric acid clearance, and plasma uric acid concentration [J]. Jama, 1991, 266(21): 3008-11.

[7] Meshkani R, Zargari M, Larijani B. The relationship between uric acid and metabolic syndrome in normal glucose tolerance and normal fasting glucose subjects [J]. Acta diabetologica, 2011, 48(1): 79-88.

[8] Khosla U M, Zharikov S, Finch J L, Nakagawa T, Roncal C, Mu W, Krotova K, Block E R, Prabhakar S, Johnson R J. Hyperuricemia induces endothelial dysfunction [J]. Kidney international, 2005, 67(5): 1739-42.

[9] Zhu Y, Hu Y, Huang T, Zhang Y, Li Z, Luo C, Luo Y, Yuan H, Hisatome I, Yamamoto T, Cheng J. High uric acid directly inhibits insulin signalling and induces insulin resistance [J]. Biochemical and biophysical research communications, 2014, 447(4): 707-14.

[10] Liu X Z, Xu X, Zhu J Q, Zhao D B. Association between three non-insulin-based indexes of insulin resistance and hyperuricemia [J]. Clinical rheumatology, 2019, 38(11): 3227-33.

[11] Han T, Lan L, Qu R, Xu Q, Jiang R, Na L, Sun C. Temporal Relationship Between Hyperuricemia and Insulin Resistance and Its Impact on Future Risk of Hypertension [J]. Hypertension (Dallas, Tex : 1979), 2017, 70(4): 703-11.

[12] Tsunoda S, Kamide K, Minami J, Kawano Y. Decreases in serum uric acid by amelioration of insulin resistance in overweight hypertensive patients: effect of a low-energy diet and an insulinsensitizing agent [J]. American journal of hypertension, 2002, 15(8): 697-701.

[13] Barazzoni R, Gortan Cappellari G, Ragni M, Nisoli E. Insulin resistance in obesity: an overview of fundamental alterations [J]. Eating and weight disorders : EWD, 2018, 23(2): 149-57. 
Ali N, Perveen R, Rahman S, Mahmood S, Rahman S, Islam S, Haque T, Sumon A H, Kathak R R, Molla N H, Islam F, Mohanto N C, Nurunnabi S M, Ahmed S, Rahman M. Prevalence of hyperuricemia and the relationship between serum uric acid and obesity: A study on Bangladeshi adults [J]. PloS one, 2018, 13(11): e0206850.

[15] Simental-Mendía L E, Rodríguez-Morán M, Guerrero-Romero F. The product of fasting glucose and triglycerides as surrogate for identifying insulin resistance in apparently healthy subjects [ $\mathrm{J}$ ]. Metabolic syndrome and related disorders, 2008, 6(4): 299-304.

[16] Minh H V, Tien H A, Sinh C T, Thang D C, Chen C H, Tay J C, Siddique S, Wang T D, Sogunuru G P, Chia Y C, Kario K. Assessment of preferred methods to measure insulin resistance in Asian patients with hypertension [J]. Journal of clinical hypertension (Greenwich, Conn), 2021, 23(3): 529-37.

[17] Shi W, Xing L, Jing L, Tian Y, Liu S. Usefulness of Triglyceride-glucose Index for estimating Hyperuricemia risk: Insights from a general Population [J]. Postgraduate medicine, 2019, 131(5): 348-56.

[18] Mazidi M, Katsiki N, Mikhailidis D P, Banach $M$. The link between insulin resistance parameters and serum uric acid is mediated by adiposity [J]. Atherosclerosis, 2018, 270(180-6.

[19] Li Y, You A, Tomlinson B, Yue L, Zhao K, Fan H, Zheng L. Insulin resistance surrogates predict hypertension plus hyperuricemia [J]. Journal of diabetes investigation, 2021,

[20] Guerrero-Romero F, Simental-Mendía L E, González-Ortiz M, Martínez-Abundis E, Ramos-Zavala M G, Hernández-González S O, Jacques-Camarena O, Rodríguez-Morán M. The product of triglycerides and glucose, a simple measure of insulin sensitivity. Comparison with the euglycemic-hyperinsulinemic clamp [J]. The Journal of clinical endocrinology and metabolism, 2010, 95(7): 3347-51.

[21] Liu R, Han C, Wu D, Xia X, Gu J, Guan H, Shan Z, Teng W. Prevalence of Hyperuricemia and Gout in Mainland China from 2000 to 2014: A Systematic Review and Meta-Analysis [J]. BioMed research international, 2015, 2015(762820.

[22] Sharaf El Din U A A, Salem M M, Abdulazim D O. Uric acid in the pathogenesis of metabolic, renal, and cardiovascular diseases: A review [J]. Journal of advanced research, 2017, 8(5): 537-48.

[23] Gois P H F, Souza E R M. Pharmacotherapy for hyperuricemia in hypertensive patients [J]. The Cochrane database of systematic reviews, 2017, 4(4): Cd008652.

[24] Verdecchia P, Schillaci G, Reboldi G, Santeusanio F, Porcellati C, Brunetti P. Relation between serum uric acid and risk of cardiovascular disease in essential hypertension. The PIUMA study [J]. Hypertension (Dallas, Tex : 1979), 2000, 36(6): 1072-8.

[25] Li X, Meng X, He Y, Spiliopoulou A, Timofeeva M, Wei W Q, Gifford A, Yang T, Varley T, Tzoulaki I, Joshi P, Denny J C, McKeigue P, Campbell H, Theodoratou E. Genetically determined serum urate levels 
and cardiovascular and other diseases in UK Biobank cohort: A phenome-wide mendelian randomization study [J]. PLoS medicine, 2019, 16(10): e1002937.

[26] Visco V, Pascale A V, Virtuoso N, Mongiello F, Cinque F, Gioia R, Finelli R, Mazzeo P, Manzi M V, Morisco C, Rozza F, Izzo R, Cerasuolo F, Ciccarelli M, laccarino G. Serum Uric Acid and Left Ventricular Mass in Essential Hypertension [J]. Frontiers in cardiovascular medicine, 2020, 7(570000.

[27] Oparil S, Acelajado M C, Bakris G L, Berlowitz D R, Cífková R, Dominiczak A F, Grassi G, Jordan J, Poulter N R, Rodgers A, Whelton P K. Hypertension [J]. Nature reviews Disease primers, 2018, 4(18014.

[28] Zoccali C, Maio R, Mallamaci F, Sesti G, Perticone F. Uric acid and endothelial dysfunction in essential hypertension [J]. Journal of the American Society of Nephrology : JASN, 2006, 17(5): 1466-71.

[29] Yu W, Cheng J D. Uric Acid and Cardiovascular Disease: An Update From Molecular Mechanism to Clinical Perspective [J]. Frontiers in pharmacology, 2020, 11(582680.

[30] Thai P V, Tien H A, Van Minh H, Valensi P. Triglyceride glucose index for the detection of asymptomatic coronary artery stenosis in patients with type 2 diabetes [J]. Cardiovascular diabetology, 2020, 19(1): 137.

[31] Kim M K, Ahn C W, Kang S, Nam J S, Kim K R, Park J S. Relationship between the triglyceride glucose index and coronary artery calcification in Korean adults [J]. Cardiovascular diabetology, 2017, 16(1): 108.

[32] Li M, Zhan A, Huang X, Hu L, Zhou W, Wang T, Zhu L, Bao H, Cheng X. Positive association between triglyceride glucose index and arterial stiffness in hypertensive patients: the China H-type Hypertension Registry Study [J]. Cardiovascular diabetology, 2020, 19(1): 139.

[33] O'Neill S, O'Driscoll L. Metabolic syndrome: a closer look at the growing epidemic and its associated pathologies [J]. Obesity reviews : an official journal of the International Association for the Study of Obesity, 2015, 16(1): 1-12.

[34] Chang S H, Yu Y C, Carlsson N P, Liu X, Colditz G A. Racial disparity in life expectancies and life years lost associated with multiple obesity-related chronic conditions [J]. Obesity (Silver Spring, Md), 2017, 25(5): 950-7.

[35] McGill J B, Haffner S, Rees T J, Sowers J R, Tershakovec A M, Weber M. Progress and controversies: treating obesity and insulin resistance in the context of hypertension [J]. Journal of clinical hypertension (Greenwich, Conn), 2009, 11(1): 36-41.

\section{Tables}


Table1 Clinical characteristics of participants by hyperuricemia

\begin{tabular}{|c|c|c|c|c|}
\hline Variables & $\begin{array}{l}\text { Total } \\
(n=4551)\end{array}$ & $\begin{array}{l}\text { Hyperuricemia } \\
(n=605)\end{array}$ & $\begin{array}{l}\text { Non-hyperuricemia } \\
(n=3946)\end{array}$ & $\begin{array}{l}\mathrm{P} \\
\text { value }\end{array}$ \\
\hline Age (year) & $58.63 \pm 8.33$ & $60.11 \pm 8.76$ & $58.41 \pm 8.24$ & $\square 0.001$ \\
\hline Male & 1531(33.6\%) & $310(51.2 \%)$ & $1221(30.9 \%)$ & {$[0.001$} \\
\hline Height (cm) & $157.70 \pm 7.94$ & $160.23 \pm 8.64$ & $157.31 \pm 7.75$ & $\square 0.001$ \\
\hline Weight (Kg) & $65.37 \pm 14.40$ & $69.80 \pm 11.22$ & $64.69 \pm 14.71$ & {$[0.001$} \\
\hline BMI $\left(\mathrm{kg} / \mathrm{m}^{2}\right)$ & $26.23 \pm 5.06$ & $27.14 \pm 3.53$ & $26.10 \pm 5.24$ & {$[0.001$} \\
\hline WC (cm) & $85.45 \pm 12.09$ & $89.68 \pm 11.92$ & $84.80 \pm 11.98$ & {$[0.001$} \\
\hline $\mathrm{HC}(\mathrm{cm})$ & $98.25 \pm 10.95$ & $100.72 \pm 10.80$ & $97.87 \pm 10.93$ & {$[0.001$} \\
\hline SBP (mmHg) & $163.50 \pm 24.47$ & $165.11 \pm 25.66$ & $163.26 \pm 24.28$ & 0.084 \\
\hline $\mathrm{DBP}(\mathrm{mmHg})$ & $97.04 \pm 12.62$ & $98.18 \pm 12.88$ & $96.86 \pm 12.57$ & 0.017 \\
\hline Glucose (mmol/L) & $5.57 \pm 1.69$ & $5.57 \pm 1.20$ & $5.56 \pm 1.75$ & 0.947 \\
\hline $\begin{array}{l}\text { Triglyceride } \\
(\mathrm{mmol} / \mathrm{L})\end{array}$ & $1.68 \pm 1.24$ & $2.17 \pm 1.52$ & $1.61 \pm 1.17$ & {$[0.001$} \\
\hline $\begin{array}{l}\text { Cholesterol } \\
\text { (mmol/L) }\end{array}$ & $5.53 \pm 1.10$ & $5.84 \pm 1.19$ & $5.48 \pm 1.08$ & {$[0.001$} \\
\hline $\mathrm{HDL}-\mathrm{C}(\mathrm{mmol} / \mathrm{L})$ & $1.55 \pm 0.34$ & $1.48 \pm 0.34$ & $1.56 \pm 0.34$ & {$[0.001$} \\
\hline LDL-C (mmol/L) & $3.15 \pm 0.86$ & $3.37 \pm 0.93$ & $3.12 \pm 0.84$ & {$[0.001$} \\
\hline BUN (mmol/L) & $5.47 \pm 1.81$ & $6.48 \pm 2.53$ & $5.31 \pm 1.61$ & $\square 0.001$ \\
\hline Creatinine(umol/L) & $66.25 \pm 26.00$ & $86.86 \pm 43.48$ & $63.09 \pm 20.37$ & $\square 0.001$ \\
\hline Uric acid (umol/L) & $292.78 \pm 86.77$ & $447.57 \pm 65.42$ & $269.05 \pm 61.56$ & {$[0.001$} \\
\hline TyG index & $8.73 \pm 0.60$ & $8.99 \pm 0.61$ & $8.70 \pm 0.59$ & 00.001 \\
\hline History of Stroke & $467(10.3 \%)$ & $83(13.7 \%)$ & $384(9.7 \%)$ & 0.002 \\
\hline History of CAD & $415(9.1 \%)$ & $85(14 \%)$ & $330(8.4 \%)$ & {$[0.001$} \\
\hline Diabetes Mellitus & $374(8.2 \%)$ & $50(8.3 \%)$ & $324(8.2 \%)$ & 0.937 \\
\hline \multicolumn{5}{|c|}{ Echocardiographic data } \\
\hline IVSd (mm) & $1.00 \pm 0.16$ & $1.03 \pm 0.16$ & $1.00 \pm 0.16$ & {$[0.001$} \\
\hline PWTd (mm) & $0.97 \pm 0.14$ & $1.00 \pm 0.14$ & $0.97 \pm 0.13$ & $\square 0.001$ \\
\hline LVM (g) & $158.65 \pm 44.11$ & $172.23 \pm 49.61$ & $156.57 \pm 42.83$ & {$[0.001$} \\
\hline
\end{tabular}


Abbreviations: BMI, body mass index; WC, waist circumference; HC, hip circumference; SBP, systolic blood pressure; DBP, diastolic blood pressure; HDL-C, high-density lipoprotein cholesterol; LDL, low-density lipoprotein cholesterol; BUN, blood urea nitrogen; CAD, coronary artery disease, TyG index, triglycerideglucose index; PWTd, end-diastolic posterior wall thickness; IVSd, end-diastolic interventricular septal thickness; $\mathrm{LVMI}_{\mathrm{h}}$, left ventricular mass index divided by height2.7; 
Table 2. Clinical characteristics of participants by TyG index

\begin{tabular}{llllll} 
Variables & Q1 $(\leq 8.32)$ & $\begin{array}{l}\text { Q2 }(8.33- \\
8.66)\end{array}$ & $\begin{array}{l}\text { Q3 }(8.67- \\
9.07)\end{array}$ & Q4 $(\geq 9.08)$ & $\begin{array}{l}\mathrm{P} \\
\text { value }\end{array}$ \\
\hline Age (year) & $\mathbf{5 8 . 5 2 \pm 8 . 7 8}$ & $58.76 \pm 8.52$ & $58.90 \pm 8.08$ & $58.35 \pm 7.93$ & 0.406
\end{tabular}

\begin{tabular}{|c|c|c|c|c|c|}
\hline Male & $474(41.6 \%)$ & $375(33.0 \%)$ & $342(30.1 \%)$ & $340(29.9 \%)$ & {$[0.001$} \\
\hline Height (cm) & $158.06 \pm 7.86$ & $157.50 \pm 8.06$ & $157.30 \pm 7.88$ & $157.94 \pm 7.93$ & 0.074 \\
\hline Weight (Kg) & $62.38 \pm 20.68$ & $64.39 \pm 11.20$ & $66.08 \pm 11.42$ & $68.65 \pm 11.15$ & $\square 0.001$ \\
\hline BMI $\left(\mathrm{kg} / \mathrm{m}^{2}\right)$ & $24.91 \pm 7.42$ & $25.90 \pm 3.17$ & $26.66 \pm 3.93$ & $27.47 \pm 3.82$ & 00.001 \\
\hline WC (cm) & $80.70 \pm 13.35$ & $84.32 \pm 11.24$ & $86.89 \pm 10.48$ & $89.92 \pm 12.09$ & $\square 0.001$ \\
\hline $\mathrm{HC}(\mathrm{cm})$ & $94.12 \pm 12.89$ & $97.84 \pm 10.18$ & $99.71 \pm 9.26$ & $101.32 \pm 9.78$ & $\square 0.001$ \\
\hline $\mathrm{SBP}(\mathrm{mmHg})$ & $160.72 \pm 24.16$ & $164.19 \pm 24.72$ & $164.18 \pm 24.52$ & $164.92 \pm 24.30$ & {$[0.001$} \\
\hline $\mathrm{DBP}(\mathrm{mmHg})$ & $95.77 \pm 12.31$ & $97.46 \pm 13.04$ & $97.00 \pm 12.43$ & $97.92 \pm 12.59$ & $\square 0.001$ \\
\hline Heart rate & $70.74 \pm 11.28$ & $72.06 \pm 11.70$ & $73.97 \pm 12.65$ & $74.51 \pm 12.99$ & $\square 0.001$ \\
\hline Glucose(mmol/L) & $4.90 \pm 0.67$ & $5.20 \pm 0.72$ & $5.46 \pm 1.00$ & $6.71 \pm 2.75$ & $\square 0.001$ \\
\hline $\begin{array}{l}\text { Triglyceride } \\
\text { (mmol/L) }\end{array}$ & $0.83 \pm 0.18$ & $1.20 \pm 0.19$ & $1.67 \pm 0.31$ & $3.03 \pm 1.78$ & $\square 0.001$ \\
\hline $\begin{array}{l}\text { Cholesterol } \\
\text { (mmol/L) }\end{array}$ & $5.05 \pm 0.90$ & $5.36 \pm 1.00$ & $5.70 \pm 1.05$ & $6.02 \pm 1.18$ & $\square 0.001$ \\
\hline $\mathrm{HDL}-\mathrm{C}(\mathrm{mmol} / \mathrm{L})$ & $1.68 \pm 0.35$ & $1.60 \pm 0.34$ & $1.52 \pm 0.32$ & $1.40 \pm 0.29$ & $\square 0.001$ \\
\hline LDL-C (mmol/L) & $2.80 \pm 0.71$ & $3.09 \pm 0.80$ & $3.36 \pm 0.87$ & $3.36 \pm 0.92$ & $\square 0.001$ \\
\hline Creatinine(umol/L) & $66.05 \pm 26.27$ & $66.67 \pm 22.48$ & $66.65 \pm 30.68$ & $65.63 \pm 23.84$ & 0.736 \\
\hline BUN (mmol/L) & $5.63 \pm 1.83$ & $5.54 \pm 1.77$ & $5.40 \pm 1.84$ & $5.30 \pm 1.76$ & 00.001 \\
\hline Uric acid (umol/L) & $267.72 \pm 75.74$ & $286.00 \pm 81.65$ & $300.58 \pm 87.62$ & $316.85 \pm 93.48$ & {$[0.001$} \\
\hline History of Stroke & $102(8.9 \%)$ & $110(9.7 \%)$ & $132(11.6 \%)$ & $123(10.8 \%)$ & 0.160 \\
\hline History of CAD & $78(6.8 \%)$ & $93(8.2 \%)$ & $95(8.4 \%)$ & $149(13.0 \%)$ & {$[0.001$} \\
\hline Diabetes Mellitus & $5(0.4 \%)$ & $17(1.5 \%)$ & $64(5.6 \%)$ & $288(25.3 \%)$ & {$[0.001$} \\
\hline Hyperuricemia & $68(6.0 \%)$ & $118(10.4 \%)$ & $175(15.4 \%)$ & $244(21.4 \%)$ & $\square 0.001$ \\
\hline
\end{tabular}

Abbreviations: BMI, body mass index; WC, waist circumference; HC, hip circumference.SBP, systolic blood pressure; DBP, diastolic blood pressure; HDL-C, high-density lipoprotein cholesterol; LDL, low-density 
lipoprotein cholesterol; BUN, blood urea nitrogen; CAD, coronary artery disease; TyG index, triglycerideglucose index.

\begin{tabular}{|c|c|c|c|c|c|c|c|c|}
\hline \multirow[t]{4}{*}{ Table 3} & \multicolumn{8}{|c|}{ Odds ratio $(95 \% \mathrm{Cl})$ of hyperuricemia by triglyceride-glucose index } \\
\hline & Q1 & & Q2 & & Q3 & & Q4 & \\
\hline & $(\leq 8.32)$ & & $(8.33-8.66)$ & & $(8.67-9.07)$ & & $(\geq 9.08)$ & \\
\hline & $\begin{array}{l}\text { OR } \\
(95 \% \mathrm{Cl})\end{array}$ & $\begin{array}{l}\mathrm{P}- \\
\text { value }\end{array}$ & $\mathrm{OR}(95 \% \mathrm{Cl})$ & $\begin{array}{l}\text { P- } \\
\text { value }\end{array}$ & $\mathrm{OR}(95 \% \mathrm{Cl})$ & $\begin{array}{l}\mathrm{P}- \\
\text { value }\end{array}$ & OR $(95 \% \mathrm{Cl})$ & $\begin{array}{l}\text { P- } \\
\text { value }\end{array}$ \\
\hline Model1 & Reference & & $\begin{array}{l}1.83(1.34- \\
2.49)\end{array}$ & $\begin{array}{l}\square \\
0.001\end{array}$ & $\begin{array}{l}2.87(2.14- \\
3.85)\end{array}$ & $\begin{array}{l}\square \\
0.001\end{array}$ & $\begin{array}{l}4.30(3.24- \\
5.71)\end{array}$ & $\begin{array}{l}\square \\
0.001\end{array}$ \\
\hline Model2 & Reference & & $\begin{array}{l}1.93(1.38- \\
2.70)\end{array}$ & 0.001 & $\begin{array}{l}3.61(2.62- \\
4.97)\end{array}$ & 0.001 & $\begin{array}{l}6.47(4.70- \\
8.91)\end{array}$ & $\begin{array}{l}\square \\
0.001\end{array}$ \\
\hline Model3 & Reference & & $\begin{array}{l}1.60(1.14- \\
2.26)\end{array}$ & 0.007 & $\begin{array}{l}2.47(1.76- \\
3.48)\end{array}$ & $\begin{array}{l}\square \\
0.001\end{array}$ & $\begin{array}{l}3.45(2.35- \\
5.07)\end{array}$ & $\begin{array}{l}0.001 \\
0.00\end{array}$ \\
\hline
\end{tabular}


Abbreviations: $95 \% \mathrm{Cl}, 95 \%$ confidence interval; OR, odds ratio.

Model 1: unadjusted

Model 2: adjusted for age, sex, systolic blood pressure, diastolic blood pressure, serum creatinine, blood urea nitrogen, the history of stroke, coronary artery disease and diabetes mellitus.

Model 3: adjusted for model 2 plus serum cholesterol, high-density lipoprotein cholesterol, low-density lipoprotein cholesterol, body mass index, waist and hip circumference.

Table 4 The association of triglyceride-glucose index index with hyperuricemia and obesity indexes

\begin{tabular}{|c|c|c|c|c|}
\hline & \multicolumn{4}{|l|}{ TyG index } \\
\hline & Q1 & Q2 & Q3 & Q4 \\
\hline & $\begin{array}{l}\beta(95 \% \mathrm{Cl}) ; \mathrm{P}- \\
\text { value }\end{array}$ & $\beta(95 \% \mathrm{Cl}) ; \mathrm{P}$-value & $\beta(95 \% \mathrm{Cl}) ; \mathrm{P}-$ value & $\beta(95 \% \mathrm{Cl})$;P-value \\
\hline BMI $\left(\mathrm{kg} / \mathrm{m}^{2}\right)$ & Reference & $\begin{array}{l}0.17(0.09-0.25) ; \gtrsim \\
0.001\end{array}$ & $\begin{array}{l}0.32(0.24-0.40) ; \gtrsim \\
0.001\end{array}$ & $\begin{array}{l}0.47(0.38-0.55) ; \bigotimes \\
0.001\end{array}$ \\
\hline WC (cm) & Reference & $\begin{array}{l}0.30(0.22-0.38) ; \rrbracket \\
0.001\end{array}$ & $\begin{array}{l}0.52(0.44-0.60) ; \rrbracket \\
0.001\end{array}$ & $\begin{array}{l}0.76(0.68-0.85) ; \nabla \\
0.001\end{array}$ \\
\hline $\mathrm{HC}(\mathrm{cm})$ & Reference & $\begin{array}{l}0.32(0.24-0.40) ; \Downarrow \\
0.001\end{array}$ & $\begin{array}{l}0.49(0.41-0.57) ; \bigotimes \\
0.001\end{array}$ & $\begin{array}{l}0.64(0.55-0.72) ; \square \\
0.001\end{array}$ \\
\hline $\begin{array}{l}\text { Uric acid } \\
\text { (umol/L) }\end{array}$ & Reference & $\begin{array}{l}0.26(0.20-0.33) ; \rrbracket \\
0.001\end{array}$ & $\begin{array}{l}0.47(0.41-0.54) ; \bigotimes \\
0.001\end{array}$ & $\begin{array}{l}0.73(0.66-0.80) ; \nabla \\
0.001\end{array}$ \\
\hline
\end{tabular}

Abbreviations: $95 \% \mathrm{Cl}$, 95\% confidence interval; TyG index, triglyceride-glucose index; BMI, body mass index; WC, waist circumference; HC, hip circumference. 
All adjusted for age, sex, systolic blood pressure, diastolic blood pressure, serum creatinine, blood urea nitrogen, the history of stroke, coronary artery disease, diabetes mellitus.

Table 5 Mediation analysis of the relationship between triglyceride-glucose index and uric acid level by obesity indexes

\begin{tabular}{|c|c|c|c|c|c|}
\hline \multirow{2}{*}{$\begin{array}{l}\text { Potential } \\
\text { mediators }\end{array}$} & \multicolumn{2}{|l|}{ Direct effect } & \multicolumn{2}{|l|}{ Indirect effect } & \multirow{2}{*}{$\begin{array}{l}\text { Proportion of } \\
\text { mediation(\%) }\end{array}$} \\
\hline & $\beta(95 \% \mathrm{Cl})$ & $\begin{array}{l}P \\
\text { value }\end{array}$ & $\beta(95 \% \mathrm{Cl})$ & $\begin{array}{l}P \\
\text { value }\end{array}$ & \\
\hline BMI & $\begin{array}{l}0.2252(0.1864- \\
0.2639)\end{array}$ & 0.001 & $\begin{array}{l}0.0178(0.0068- \\
0.0382)\end{array}$ & 0.001 & $7.3 \%$ \\
\hline WC & $\begin{array}{l}0.2021(0.1632- \\
0.2411)\end{array}$ & $\begin{array}{l}\square \\
0.001\end{array}$ & $\begin{array}{l}0.0408(0.0310- \\
0.0518)\end{array}$ & $\stackrel{\square}{\square} 001$ & $16.7 \%$ \\
\hline $\mathrm{HC}$ & $\begin{array}{l}0.2181(0.1792- \\
0.2570)\end{array}$ & 0.001 & $\begin{array}{l}0.0249(0.0180- \\
0.0327)\end{array}$ & ${ }^{\square} 0.001$ & $10.2 \%$ \\
\hline
\end{tabular}

Abbreviations:OR, Odd ratio. 95\% Cl, 95\%Confidence interval; TyG index, triglyceride-glucose index; BMI, body mass index; WC, waist circumference; $\mathrm{HC}$, hip circumference.

Adjusted for age, sex, systolic blood pressure, diastolic blood pressure, serum creatinine, blood urea nitrogen, the history of stroke, coronary artery disease and diabetes mellitus, serum cholesterol, highdensity lipoprotein cholesterol, low-density lipoprotein cholesterol.

\section{Figures}



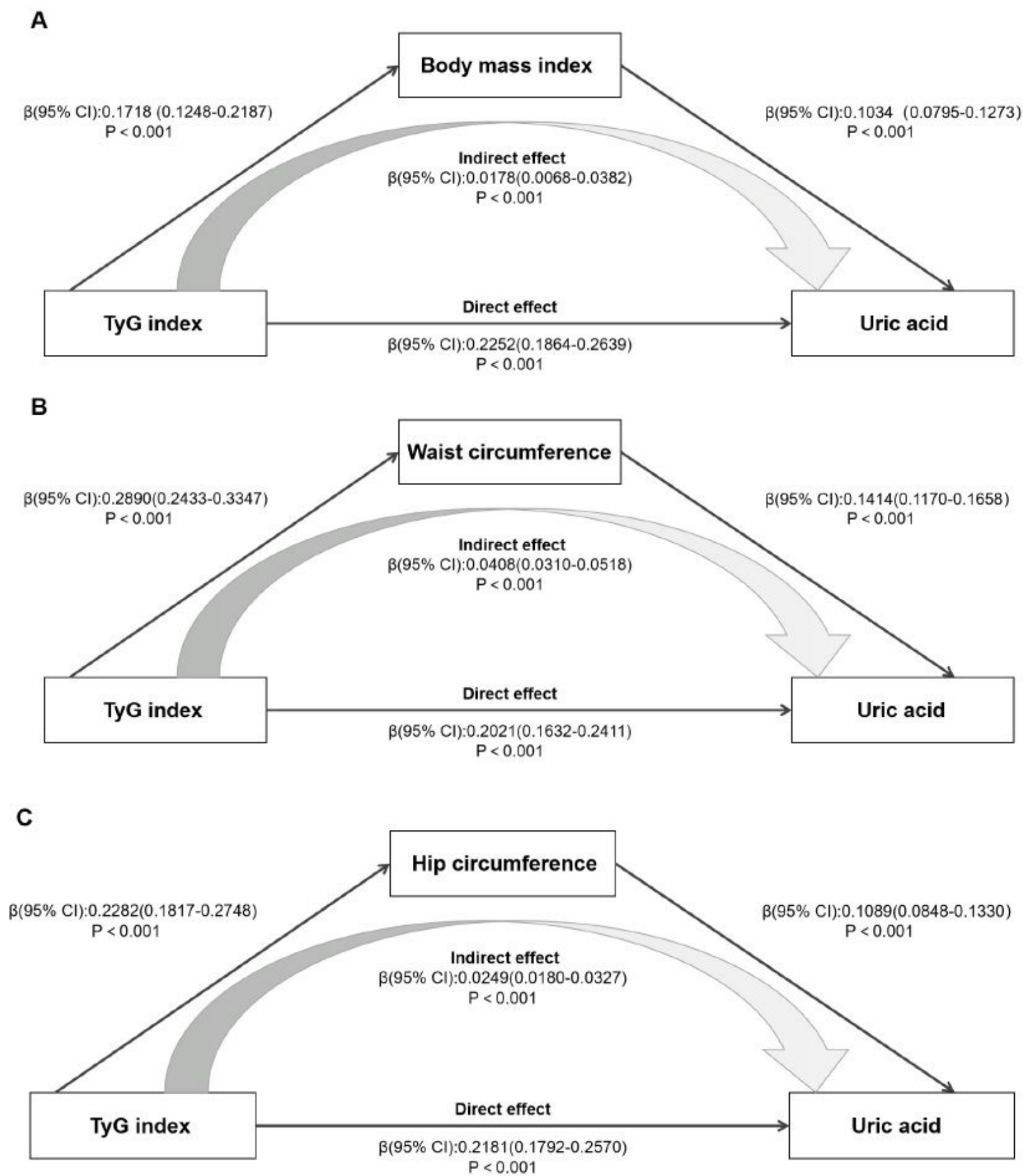

\section{Figure 1}

Mediation effect to BMI (A) or WC (B) or HC (C) on the relationship between TyG index and uric acid. The parameter estimate of total effect is $0.2430(0.2041-0.2818), P \otimes 0.001$. Adjusted for age, sex, systolic blood pressure, diastolic blood pressure, serum creatinine, blood urea nitrogen, the history of stroke, coronary artery disease and diabetes mellitus, serum cholesterol, high-density lipoprotein cholesterol, low-density lipoprotein cholesterol. 